\title{
Ongoing outbreak of Shigella flexneri serotype 3a in men who have sex with men in England and Wales, data from 2009-2011
}

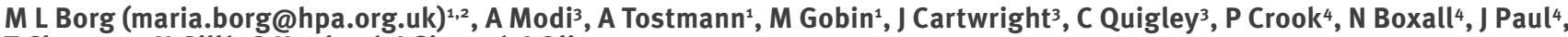

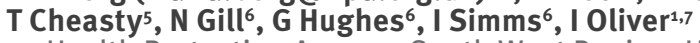

1. Health Protection Agency, South West Region, United Kingdom

2. European Programme for Intervention Epidemiology Training (EPIET), European Centre for Disease Prevention and Control (ECDC), Stockholm, Sweden

3. Health Protection Agency, North West Region, United Kingdom

4. Health Protection Agency, South East Region, United Kingdom

5. Gastro-Intestinal Infections Reference Unit, Health Protection Agency - Colindale, London, United Kingdom

6. HIV \& STI Department, Health Protection Agency - Colindale, London, United Kingdom

7. University of Bristol, Bristol, United Kingdom

Citation style for this article:

Borg ML, Modi A, Tostmann A, Gobin M, Cartwright J, Quigley C, Crook P, Boxall N, Paul J, Cheasty T, Gill N, Hughes G, Simms I, Oliver I. Ongoing outbreak of

Shigella flexneri serotype $3 a$ in men who have sex with men in England and Wales, data from 2009-2011.

Euro Surveill. 2012;17(13):pii=20137. Available online: http://www.eurosurveillance.org/ViewArticle.aspx?Articleld=20137

Article published on 29 March 2012

Diagnoses of Shigella flexneri in the United Kingdom (UK) are usually travel-related. However, since 2009, there has been an overall increase in UK-acquired cases. The Health Protection Agency has been investigating a national outbreak of $S$. flexneri detected in 2011 and which is still ongoing. Cases occurred mostly in men who have sex with men and were of serotype 3a. The investigation aimed at obtaining epidemiological data to inform targeted outbreak management and control.

Cases of Shigella flexneri in the United Kingdom (UK) usually originate from travel or contact with travellers from higher incidence regions such as Indian subcontinent, North and East Africa and South America [1]. Following analyses of laboratory data, an increase in UK-acquired S. flexneri cases was detected in London in November 2010. A subsequent rise in UK-acquired cases was also noted in Manchester in May 2011. The initial cases reported were predominantly of serotype $3 a$ and mostly among men who have sex with men (MSM) aged between 30 and 50 years, some of whom were HIV positive. Pulsed field gel electrophoresis (PGFE) performed on initial stool specimen showed that some of the isolates were indistinguishable, however preliminary investigation failed to identify a common venue or point source $[2,3]$.

In response, a national outbreak control team was formally established in September 2011 to investigate and manage the outbreak of S. flexneri. Enhanced surveillance was initiated in order to:

- describe the epidemiology of S. flexneri infection in individuals who had no travel history or who had travelled to countries with low risk for infection;
- estimate the proportion of UK-acquired cases or cases associated with travel in low-risk countries that are explained by transmission in MSM;

- identify risk factors for transmission of S. flexneri between MSM.

Sexual transmission of Shigella was first described in the United States during the 1970s [4]. Since then, several outbreaks of sexually transmitted Shigella, predominantly in MSM, have been reported [5-8]. In 2006, an outbreak of Shigella among MSM in London coincided with a similar outbreak in Berlin suggesting that travel plays a role in introducing Shigella species to populations at risk $[9,10]$.

\section{Outbreak investigation}

National enhanced surveillance of S. flexneri was conducted from September to December 2011 inclusive, in order to describe and monitor the epidemiology of the outbreak. The population under surveillance consisted of UK-acquired $S$. flexneri infection cases and reported cases associated with travel in low-risk countries.

Low-risk travel-associated individuals were defined as individuals who returned to the UK in the four days before onset of illness after travel to countries with low risk for Shigella infection (Europe, North America and Australia). High-risk travel-associated diagnoses were defined as individuals who returned to the UK in the four days before onset of illness after travel to countries with high risk for Shigella infection (South America, Asia and Africa) [1].

A confirmed case was defined as a laboratoryconfirmed case of $S$. flexneri with a specimen date 
between 1 September and 31 December 2011 with no recent travel or who reported recent travel to low-risk countries.

A probable case was defined as a laboratory-confirmed case of $S$. flexneri with an unknown travel history.

Cases of S. flexneri among people who had travelled to high-risk countries or secondary cases of $S$. flexneri who were contacts of high risk travel-associated cases were excluded.

All laboratories were asked to notify Shigella isolations and to send stool specimens to the national reference laboratory (Gastrointestinal Infections Reference Unit, Health Protection Agency - Colindale, London) for serotyping, PFGE analysis and sensitivity testing. Weekly updates on laboratory-confirmed S. flexneri diagnoses were forwarded to the respective regions for further follow-up.

Local health protection units confirmed the travel history for every reported S. flexneri diagnosis and conducted an interview using a surveillance questionnaire for UK-acquired or low-risk travel-associated diagnoses of $S$. flexneri. The questionnaire contained additional questions on exposures such as travel, food history, contact with symptomatic individuals and sexual contact to assist with case management. In-depth interviews with confirmed MSM cases were also conducted to identify potential risk factors for infection.

S. flexneri reports from the national laboratory databases, regions and local units were collected and analysed and feedback was disseminated to the regional units and identified leads through epidemiological update reports.

Increased awareness and guidance for health professionals and people at risk of infection was issued through HPA briefings, information leaflets and press releases [11].

S. flexneri diagnoses reported by the national laboratories between 2001 and 2011 were also analysed to provide context to the current outbreak and to produce historical time trends.

\section{Results}

During the enhanced surveillance period between September and December 2011, 145 S. flexneri diagnoses were reported of which 37 (25.5\%) were nontravel related. Thirty-one cases were confirmed as being UK-acquired whereas six reported diagnoses were likely to be secondary cases linked to a symptomatic contact with recent travel to a high-risk country.

Eighty-six cases (59.3\%) were associated with travel to high-risk countries and the travel history was unknown for 22 individuals (15.2\%). No low-risk travel-associated cases of $S$. flexneri were reported during the enhanced surveillance period.

The UK-acquired cases were predominantly male $(n=26)$ whereas travel-associated S. flexneri diagnoses were equally distributed between both sexes: $48 \%$ male $(n=40)$ and $52 \%$ female $(n=43)$ as shown in Figure 1. The sex and age of three travel-associated cases was not known.

\section{FIGURE 1}

Cases of Shigella flexneri reported during the enhanced surveillance period by age group and sex, England and Wales, September - December 2011

A

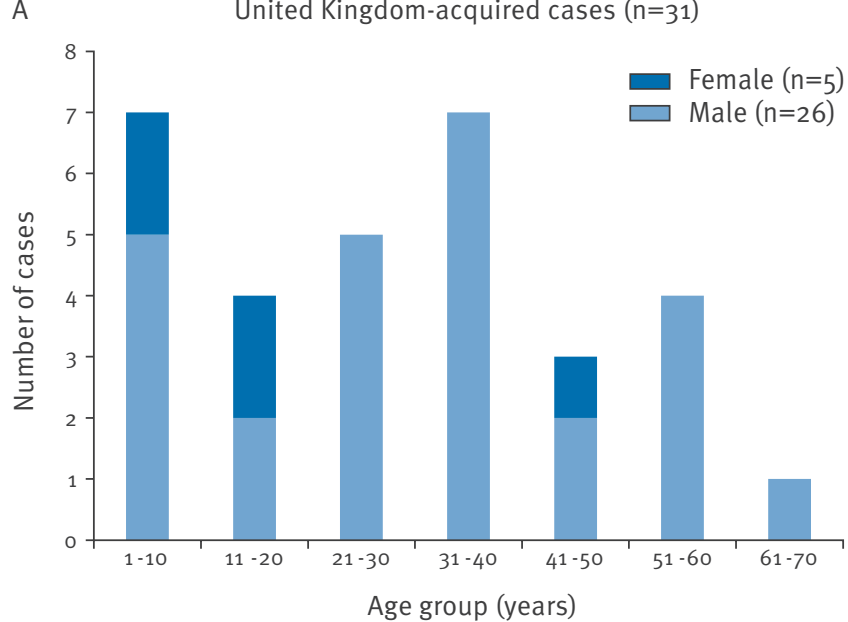

$\mathrm{B}$

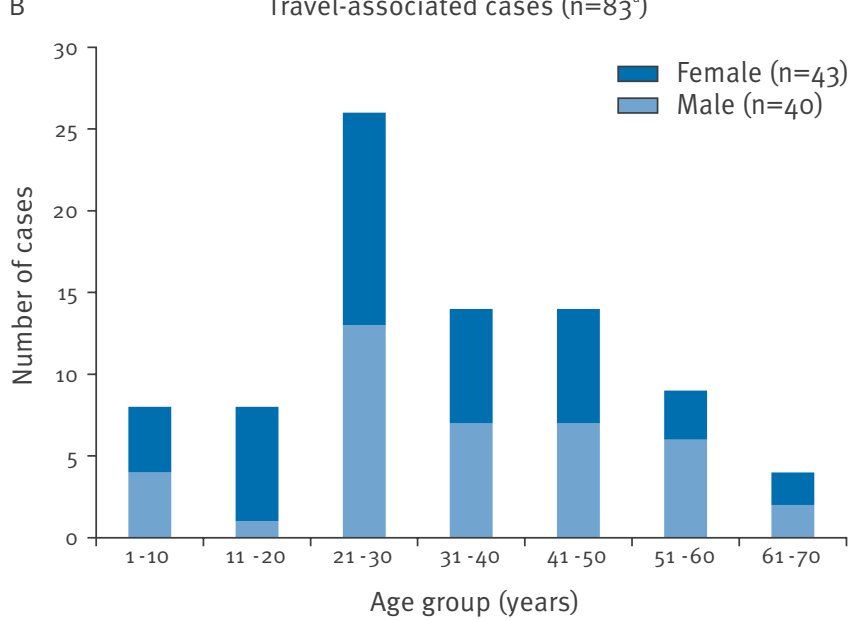

a The gender and age of three travel-associated cases was not known.

Source: National reference laboratory database (GDW- Gastro Data Warehouse), Health Protection Agency, Colindale, United Kingdom. National laboratory reporting database (LabBase 2), Health Protection Agency, Colindale, United Kingdom. 
Eleven male cases with UK-acquired S. flexneri reported MSM activity in the week before developing gastroenteritis. Three individuals refused to disclose their sexual orientation.

Ten of the 31 reported UK-acquired S. flexneri cases were serotype $3 a$, seven were serotype $1 b$, five were serotype $2 a$, three were serotype 6 and one case was reported for serotypes $1 \mathrm{a}, 1 \mathrm{c}, 2 \mathrm{~b}$ and $3 \mathrm{~b}$. The serotype was unknown for two reported $S$. flexneri diagnoses. More than half $(n=5)$ of the infections in MSM were caused by serotype $3 a$, four by serotype $1 b$, one by serotype $2 \mathrm{a}$ and one by serotype 6 .

\section{FIGURE 2}

Shigella flexneri cases with no or unknown travel history stratified by year and sex, England and Wales, 2001-2011 $(\mathrm{n}=3,352)$

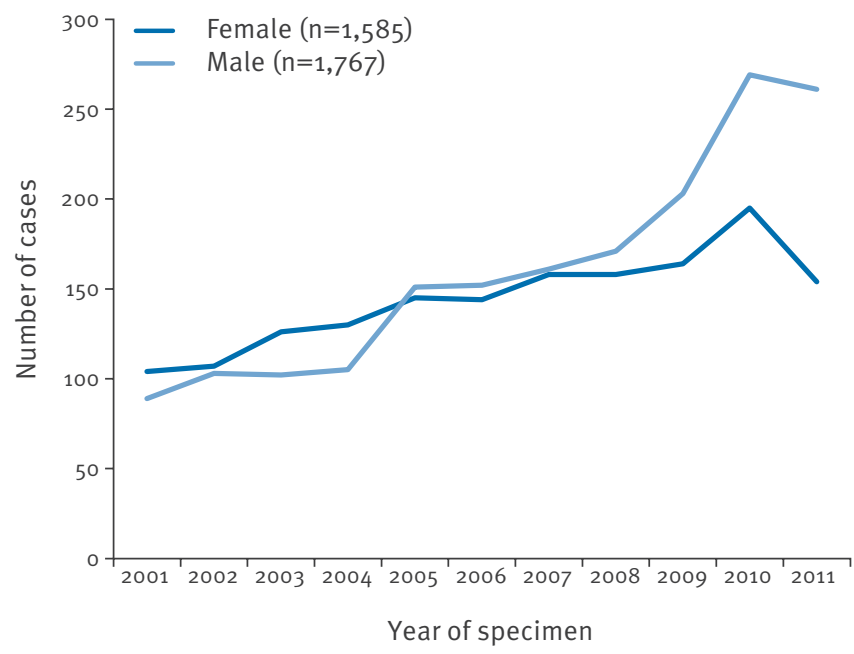

Source: National laboratory reporting database (LabBase 2), Health Protection Agency, Colindale, United Kingdom.
In depth interviews with seven MSM cases showed that they all had one long term partner and attended regular medical examinations. However, all cases reported having a casual sexual partner in the week preceding illness. These interviews revealed lack of awareness about Shigella and of the risks associated with unprotected oral and oral-anal sex.

Trends in S. flexneri diagnoses reported between 2001 and 2011 showed a gradual increase in the number of cases with no or unknown history of travel since 2001, with a similar trend in both sexes until 2008 (Figure 2). However, from 2009 onwards, numbers of diagnoses rose far more rapidly in men (Figure 2 ).

Data analysis revealed similar trends in cases between sexes and within the same age group, however, since 2009 the increase in the number of $S$. flexneri cases reported was attributable to an overrepresentation of men aged between 31 and 50 years (Figure 3).

Figure 4 shows the number of $S$. flexneri diagnoses by serotype from 2004 to 2011 . The number of cases infected with serotype 3 a has increased considerably and as from 2009 it has become as predominant as the 2 a serotype and accounted for the increase in S. flexneri cases between 2009 and 2011.

The increase in serotype $3 a$ since 2009 was mostly attributable to diagnoses among men aged 30-50 years which constituted $65 \%(211 / 324)$ of all S. flexneri 3a reports with no or unknown travel history between 2009 and 2011. When focusing on the male adult cases with serotype $3 a$, the number of monthly S. flexneri diagnoses in 2007/2008 fluctuates between 1 and 7 cases. The number of monthly reports increases to between 5 and 15 from 2009 onwards. The following graph shows the number of monthly diagnoses from

\section{FIGURE 3}

Shigella flexneri cases by sex and age group, England and Wales, (A) 2001-2008 (n=2,026) and (B) 2009-2012 (n=1,239)
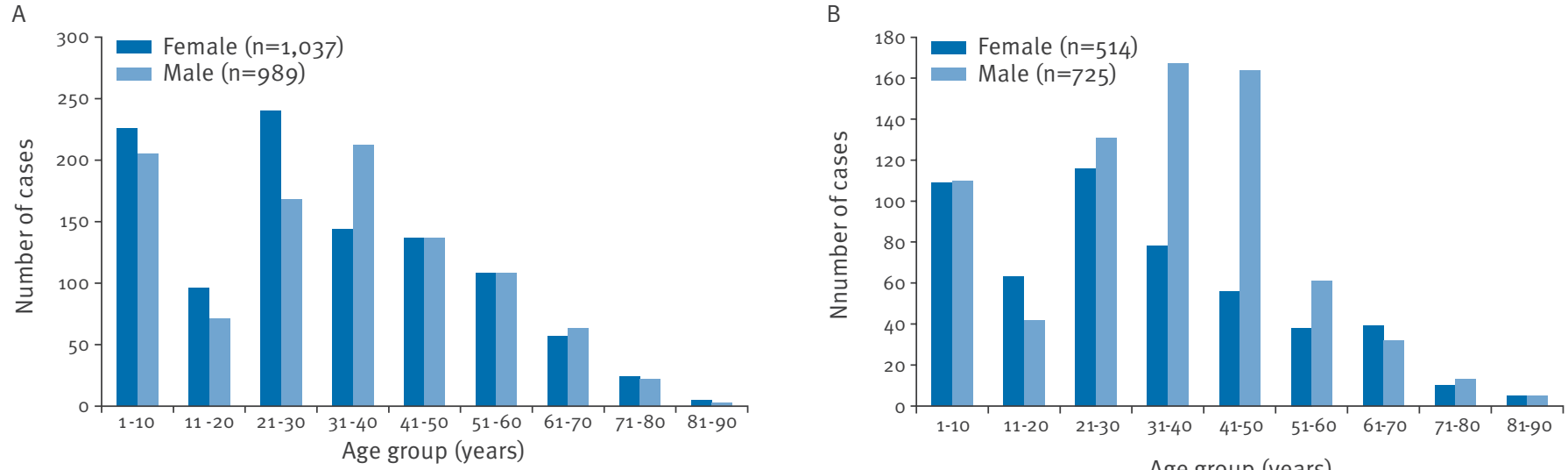

Source: National laboratory reporting database (LabBase 2), Health Protection Agency, Colindale, United Kingdom. 
2007-2012 and a three-month moving average (Figure 5).

\section{Control measures}

The outbreak control team introduced control measures which focused on actions aimed at prompt and effective management of cases to prevent onward transmission. They included increasing awareness among clinicians and MSM and prompt diagnosis and treatment, increased testing of MSM with diarrhoea and treatment of laboratory-confirmed cases with ciprofloxacin [12] subject to antimicrobial sensitivity.

\section{FIGURE 4}

Shigella flexneri serotype by year of report for cases with $S$. flexneri infection with no or unknown travel history,

England and Wales, 2004-2011 $(n=2,350)$

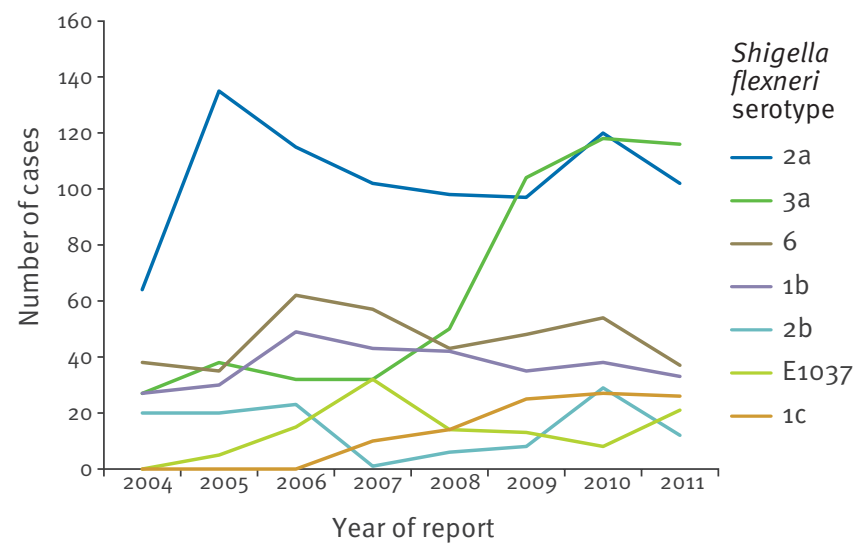

Source: National reference laboratory database (GDW- Gastro Data Warehouse), Health Protection Agency, Colindale, United Kingdom.
These actions also included recommendations regarding behaviours that may contribute to prevent further transmission:

- wash hands after using toilet, before preparing or eating food and after sexual activity;

- avoid anal sex, oral-anal sex, scat and rimming whilst symptomatic and until test for infection shows clearance;

- use of condoms, gloves, dental dams during sex;

- avoid sharing douching materials and sex toys;

- avoid swimming pools and spa centres whilst ill and for two weeks after recovery.

Work is ongoing to identify risk factors for infection and evaluate other possible control measures such as screening of asymptomatic contacts.

\section{Discussion and conclusion}

As the outbreak is still ongoing and no similar S. flexneri outbreaks have recently been reported by other countries, increased vigilance and monitoring by other European countries is recommended in order to promptly and effectively detect any change in the reported trends of $S$. flexneri.

Although some people may have been reluctant to disclose details about their sexual orientation, the enhanced surveillance revealed a strong association between UK-acquired $S$. flexneri and transmission in MSM. The outbreak will continue to be monitored through routine arrangements and information on cases occurring in MSM will continue to be collected in order to effectively describe the epidemiology of the disease in MSM and identify any potential risk factors to inform public health action.

\section{FIGURE 5}

Adult male cases of Shigella flexneri 3a infection with no or unknown travel history, England and Wales, January 2007January $2012(\mathrm{n}=381)$

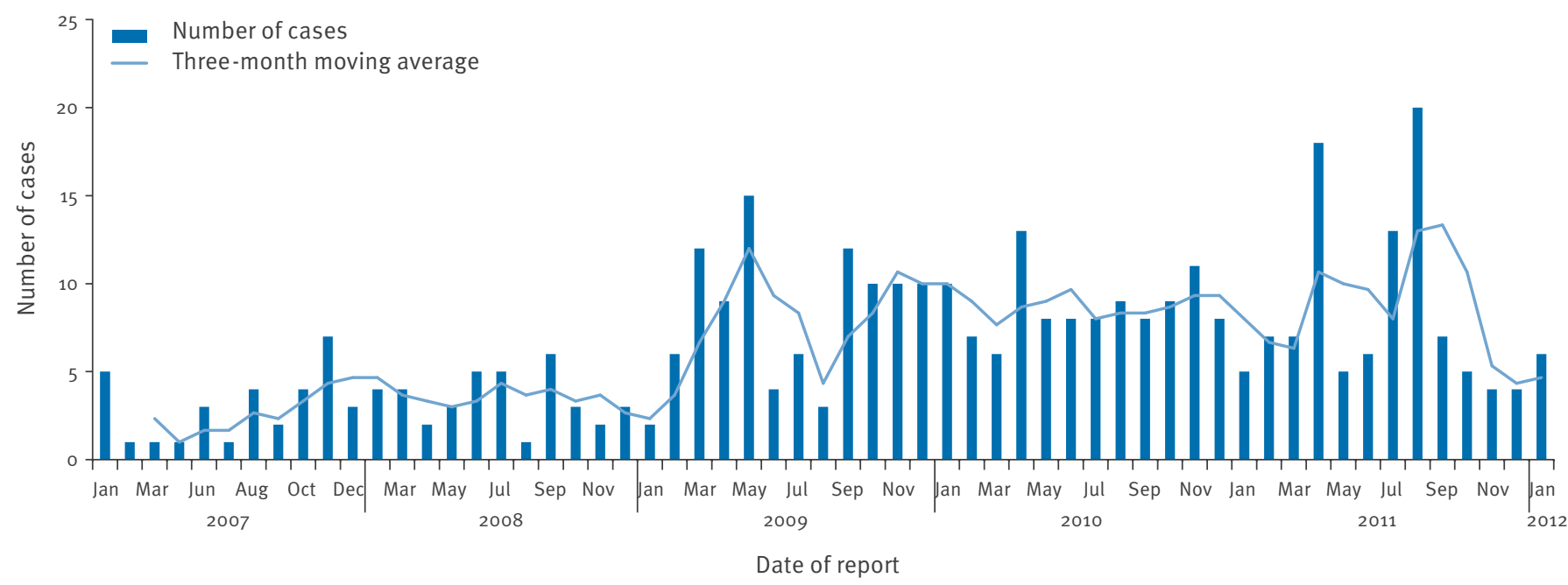

Source: National reference laboratory database (GDW- Gastro Data Warehouse), Health Protection Agency, Colindale, United Kingdom. 
Although the $S$. flexneri outbreak first emerged in 2009 and has been sustained since then, it has only been detected relatively recently. An evaluation of Shigella infection surveillance will therefore be carried out in order to identify factors leading to the delay in outbreak identification and to explore new approaches to routine surveillance of sexually-transmitted Shigella infection.

\section{References}

1. Kotloff K, Winickoff J, Ivanoff B, Clemens J, Swerdlow D, Sansonetti P, et al. Global burden of Shigella infections: implications for vaccine development and implementation of control strategies. Bull World Health Organ. 1999;77(8):651-66.

2. Health Protection Agency (HPA). Outbreak of Shigella flexneri in men who have sex with men. Health Protection Report; 5(40). 7 Oct 2011. Available from: http://www.hpa.org.uk/hpr/ archives/2011/newS4011.htm\#shgflx

3. Health Protection Agency (HPA). Outbreak of UK acquired Shigella flexneri in men who have sex with men: an update. Health Protection Report; 5(48). 2 Dec 2011. Available from: http://www.hpa.org.uk/hpr/archives/2011/news4811. htm\#shgflxnr

4. Dritz SK, Back AF. Shigella enteritis venereally transmitted. N Engl J Med. 1974;291(22):1194.

5. Marcus U, Zucs P, Bremer V, Hamouda O, Prager R, Tschaepe $\mathrm{H}$, et al. Shigellosis-a re-emerging sexually transmitted infection: outbreak in men having sex with men in Berlin. Int J STD AIDS. 2004;15(8):533-7.

6. O'Sullivan B, Delpech V, Pontivivo G, Karagiannis T, Marriott D, Harkness J, et al. Shigellosis Linked to Sex Venues, Australia. Emerg Infect Dis. 2002;8(8):862-4.

7. Centers for Disease Control and Prevention (CDC). Shigella sonnei outbreak among men who have sex with men-San Francisco, California, 2000-2001. MMWR Morb Mortal Wkly Rep. 2001;50(42):922-6.

8. Outbreak of Shigella flexneri and Shigella sonnei enterocolitis in men who have sex with men, Quebec, 1999 to 2001. Can Commun Dis Rep. 2005;31(8):85-90.

9. Drusin LM, Genvert G, Topf-Olstein B, Levy-Zombek E. Shigellosis. Another sexually transmitted disease? Br J Vener Dis. $1976 ; 52(5): 348-50$.

10. Morgan O, Crook P, Cheasty T, Jiggle B, Giraudon I, Hughes $\mathrm{H}$, et al. Shigella sonnei Outbreak among Homosexual Men, London. Emerg Infect Dis. 2006;12(9):1458-60.

11. Health Protection Agency (HPA). HPA Issues Warning About an Outbreak of Shigella Flexneri dysentery in Men Who Have Sex with Men. London: HPA. 7 Oct 2011. Press release. Available from: http://www.hpa.org.uk/NewsCentre/NationalPressRelea ses/2011PressReleases/111007ShigellaFlexneri/

12. World Health Organization (WHO). Guidelines for the control of shigellosis including epidemics due to Shigella dysenteriae Type 1. Geneva: WHO; 2005. Available from: http://whqlibdoc. who.int/publications/2005/9241592330.pdf 